\title{
Guest Editorial: The Metaphor of Paris
}

\author{
YVES AGID
}

There is a looming gap among social scientists and neuroscientists in general as far as mental functions are concerned. Although we may agree on the definition of mental functions such as "consciousness," "memory," and "language," we only understand up to a certain extent how the human brain is able to generate and control these functions. Why do we talk? Why are we able to have a memory? Why are we able to implement strategies and actions? Although we have increased our knowledge of the mechanisms of mental abilities, especially over the last 20 years, we are only beginning to find answers to these questions. This is particularly true regarding the various nervous circuits, that is, the routes used to produce our thoughts and emotions. We are starting to understand how the myriad of information from the environment arrives in the brain and how it leaves through actions expressed by movements. But, how all the information coming from the environment or produced internally is processed in the brain is still a matter of discovery, probably because our research methods are currently insufficient, either too rough in a large volume of brain tissue (neuroimagery) or too precise in a restricted area of nervous tissue (cellular biology).

A characteristic difficulty for cognitivists is the all too numerous models of brain functioning, so far based only on the description of neurons. Indeed, among the different neuronal circuits that elicit information to travel through the brain, some are like large routes (such as the pathways originating from the cerebral cortex and projecting to the spinal cord and muscles), and others are minuscule (such as the numerous inter-neurons distributed through the entire nervous system). However, neurons represent less than half of the cells in the nervous system. What about the other half of the brain? These non-neuronal cells are called "glial cells." The word comes from "glue," as, in the past, these cells were considered to constitute a sort of cement between neurons, allowing neurons to stay within the volume of the skull. This is no longer held to be the case as glial cells are now divided into several categories, the metabolisms and functions of which are even more sophisticated than those of neurons.

A way to illustrate our understanding of the respective role of neurons and glial cells in order to explain how a material brain produces immaterial thoughts (odorless, tasteless, senseless, unseen-although they can be heard) is to refer to the "Metaphor of Paris." Imagine somewhere on a planet far away, there is a scientist with an astronomical telescope so powerful that he is able to focus on minute details. He looks around at various stars and planets and chooses a certain planet called Earth.

Since our interplanatary scientist is very rigorous and thorough, he decides to put his full attention on one specific place and he zooms in on Paris. He narrows his selection further to one street and distinguishes a moving vehicle with "Milk Delivery" written on its side. He decides to follow it and records in his notebook 


\section{Guest Editorial}

that the vehicle takes the same trajectory every 12-hour period. It leaves the same building every morning to deposit small bottles filled with a white liquid and returns in the evening. The vehicle never deviates from the trajectory, passing through the same streets, and following the same pattern.

Our scientist decides to write in his notebook that on this distant planet, he has discovered an object that repeats the same complicated trajectory every day at a fixed time. The pattern can be measured by a marker, namely the vehicle. These repetitive activities occur as if the communication channel had learning abilities and therefore memory potentialities. As he confirms this initial observation by examining other vehicles using other trajectories, he writes in his lab notes:

First, I have observed that the vehicle is always making the same trajectory in Paris. There appears to be an interesting way of communication as if it has a sort of memory that allows this vehicle to always adopt the same trajectory.

Second, these communication routes are surrounded on either side by big buildings where I see creatures entering and leaving. I do not know the purpose of these edifices. Would it be possible that cultural, economic, and political decisions that allow the city to survive are taken in these cubes? My measuring instrument cannot answer the question.

And because the scientist is very modest, he makes a notation: "Unfortunately, I have only been able to observe with my telescope what is apparent and that leaves me with a very superficial concept of what is going on. I cannot see what is happening in the buildings or under the streets. To know more, I would have to go into the buildings and dig under the streets."

If we transpose the Metaphor of Paris to the brain, one can imagine that in the brain, the streets that provide communications are the neurons and that the houses that surround the streets are the glial cells (essentially "astrocytes" that represent most of the glial cells). Would it not be in these glial cells that the processes responsible for the mental functioning, that we call thoughts, takes place and lead to the behavior of living beings-whether those thoughts be words, actions, emotions, or abstractions?

Nevertheless, it is important to keep in mind that, if we are interested in scientifically understanding the bases of the functioning of the human brain, it is necessary to take into account not only the cybernetics of neurons but also the complex functions of the other half of the brain composed of glial cells. And, if we accept this challenge the consequences should be crucial in terms of our ability to model brain functioning, comprehend brain physiology, and manage patients with diseases of the nervous system. 